\title{
FAROS-E: a compact and low-cost millimeter wave surveillance radar for real time drone detection and classification
}

\author{
Samiur Rahman*, Duncan A. Robertson* \\ "University of St Andrews \\ St Andrews, Scotland \\ email: sr206@st-andrews.ac.uk
}

\begin{abstract}
This paper describes the system architecture and performance of the FAROS-E $77 \mathrm{GHz}$ radar designed and built at the University of St Andrews for real time drone detection and classification (drone vs non-drone). The motivation behind the system is to demonstrate a highly reliable drone classification sensor for security surveillance in a compact, low-cost and potentially portable form factor. The low phase noise, coherent architecture exploits the high Doppler sensitivity available at millimeter wave frequencies, to enable robust micro-Doppler signature analysis and classification. Outdoor trials of FAROS-E have yielded high fidelity data and shown very promising results as a shortrange system capable of detecting a DJI Phantom Standard 3 up to $\sim 300 \mathrm{~m}$ and classifying it up to $\sim 150 \mathrm{~m}$. The classification algorithm is capable of classifying the presence of a drone even when it is hovering stationary.
\end{abstract}

\section{Introduction}

The current trend in the market of affordable commercial drones has brought various security threats along with the socio-economic advantages of this rapidly developing technology [1]. To tackle this, radar sensors have been explored quite extensively in recent years due to their ability to detect and locate targets irrespective of daylight or weather conditions. One of the main advantages of a radar sensor is that it can obtain a characteristic micro-Doppler signature imparted by the rotation of the drone propeller blades [2], [3]. Micro-Doppler based drone classification techniques have been quite thoroughly reported in the literature [4]-[7]. Based on this approach, numerous radar systems dedicated to drone detection have been developed and commercialized [8], [9].

In some cases, drone detection radar systems are derived from existing military radars and operate at X-band or below. Whilst they may offer long detection ranges out to several kilometers, for many applications this is unnecessarily far and the systems are large, heavy and expensive. A stakeholder survey revealed that there is a demand for small, possibly portable, and more affordable radar systems to counter drone threats and users would be happy to accept more modest detection ranges e.g. in urban environments where lines of short are typically short. These could be used as part of an ad hoc network of other sensors, providing flexibility to the overall security system. To address this requirement, the Millimeter Wave Group at the University of St Andrews has designed, assembled and tested an E-band radar system demonstrator with a compact, low-cost architecture capable of detecting and classifying drones from non-drones over ranges of a few hundred meters. In this paper, a detailed description of the overall system design and performance is provided. 


\section{System design and link budget}

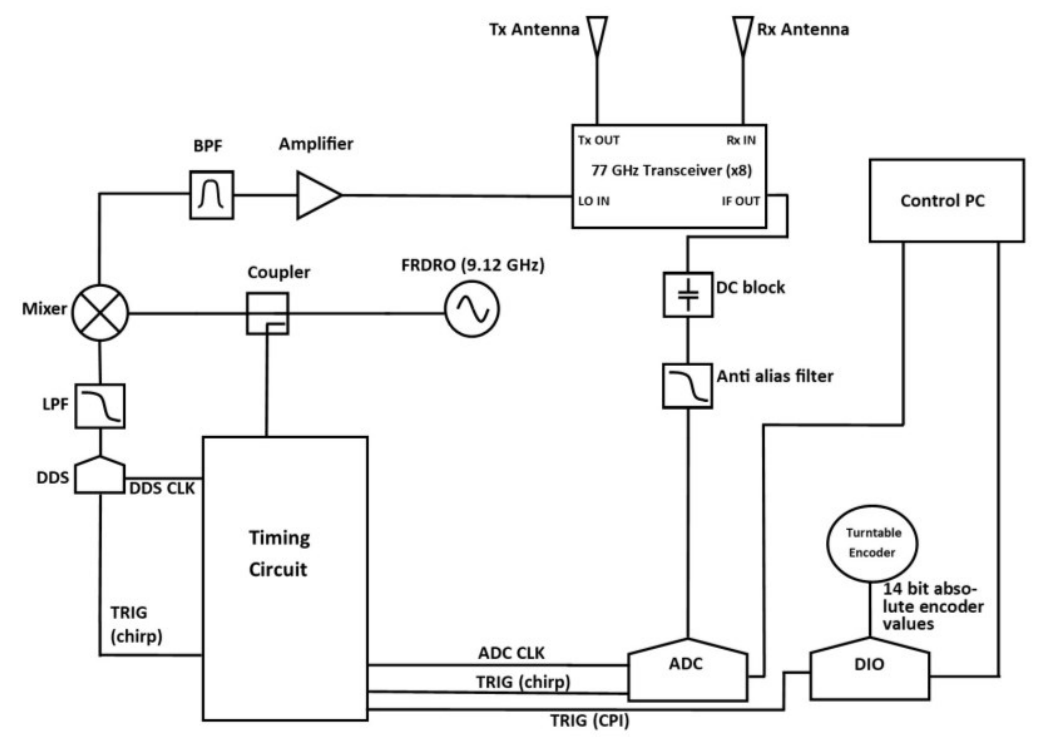

Figure 1. Block diagram of the $77 \mathrm{GHz}$ drone classification radar, FAROS-E

The major advantages of developing a millimeter wave radar system for drone classification are that it will require smaller antennas, hence reducing the size and weight of the system, whilst providing high fidelity Doppler signatures [10]. The operating frequency of $77 \mathrm{GHz}$ was chosen due to the availability of commercially available chipsets produced for the automotive industry. Fig. 1 shows the block diagram of FAROS-E, which is a dual-antenna, homodyne frequency modulated continuous wave (FMCW) system. Chirps are generated with an Analog Devices AD9914 direct digital synthesizer (DDS), upconverted onto a local oscillator (LO) and then multiplied (x8) by the transceiver to obtain the $77 \mathrm{GHz}$ signal.

In Fig. 1, it can be seen that a single free-running dielectric resonator oscillator (FRDRO) producing $9.12 \mathrm{GHz}$ is used to derive all the frequencies, including the DDS clock, ADC clock and chirp trigger, thus making the radar fully coherent for Doppler processing. The front end is a commercially available $77 \mathrm{GHz}$ transceiver manufactured by Farran Technology [11]. The transmit power is $+25 \mathrm{dBm}$ and the receiver gain is $\sim 33 \mathrm{~dB}$ in the range $76-77 \mathrm{GHz}$. The noise figure ranges from 4-6 dB at 0-12 $\mathrm{MHz}$ IF frequency values.

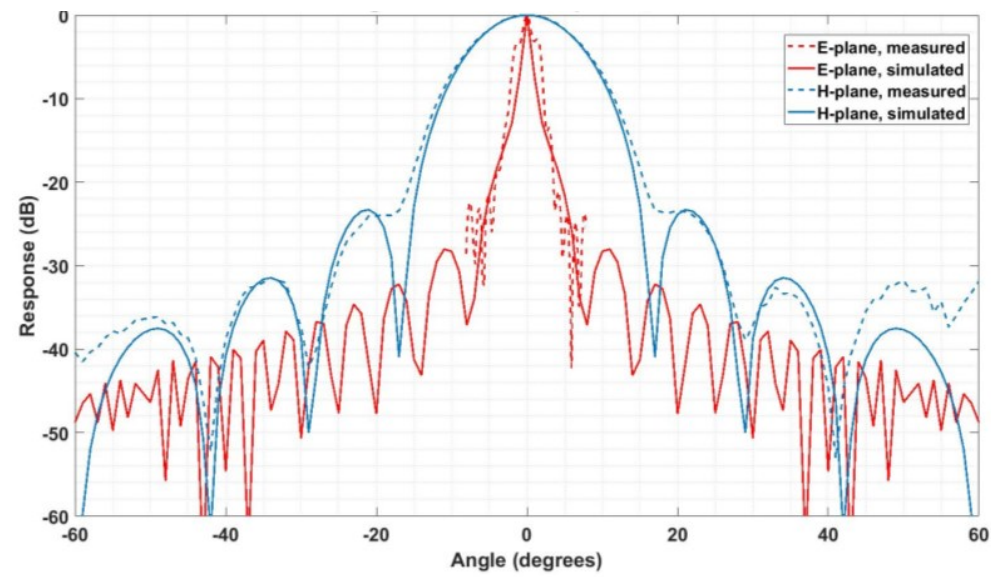

Figure 2. Antenna beam patterns of the $77 \mathrm{GHz}$ sectoral lens horn antenna

Two E-band sectoral lens horn antennas were acquired from Eravant (formerly Sage Millimeter Technologies) to provide horizontally polarized elevation fan beam antenna patterns for 
transmit and receive. The quoted gain of the antenna units was $33 \mathrm{dBi}$ at $77 \mathrm{GHz}$, with azimuth (E-plane) and elevation (H-plane) beamwidths of $1.3^{\circ}$ and $10.4^{\circ}$ respectively. The antenna patterns were measured in an outdoor test range at a distance of $38 \mathrm{~m}$ which was greater than the far-field distance of $21 \mathrm{~m}$, for performance verification and the results are shown in Fig. 2 compared with the manufacturer's predictions. It can be seen that the $3 \mathrm{~dB}$ azimuth (E-plane) beamwidth is slightly wider than predicted $\left(\sim 2.3^{\circ}\right)$ and there is some distortion of the mainlobe shape but the general agreement is acceptable. It is suspected that the ripples on the beampattern are due to imperfections in the correcting lenses. The elevation (H-plane) pattern is in good agreement with the design. The boresight gain was measured with respect to a standard gain horn and found to be $\sim 1.4 \mathrm{~dB}$ lower than the specified value. The radar head rotates continuously in azimuth to provide $360^{\circ}$ surveillance and the antennas are tilted upwards to illuminate a sector in elevation.

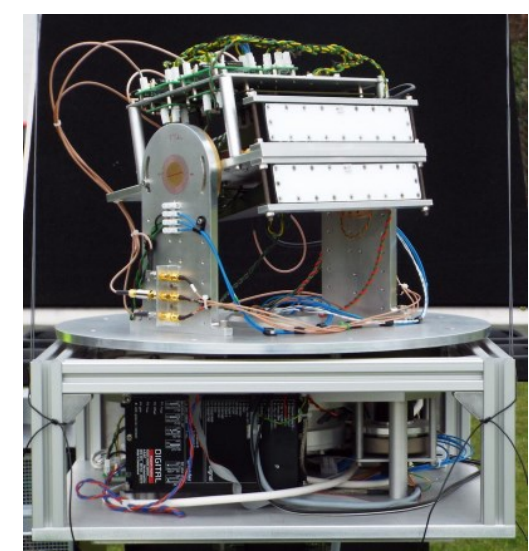

Figure 3. FAROS-E radar system

The chirp, data acquisition and scanning parameters were chosen by considering various tradeoffs between dwell time, number of samples, coherent processing interval (CPI), maximum range etc. An Adlink 9846D ADC card is used with the sampling clock derived in hardware as $17.8 \mathrm{MHz}$, giving a maximum IF frequency value of $8.9 \mathrm{MHz}$. The chirp time is $57.48 \mu$ s and the chirp period is $59.28 \mu \mathrm{s}$. A total of 64 contiguous chirps are used for a single CPI $(3.79 \mathrm{~ms})$. Two CPIs per beam were chosen to achieve sufficient dwell on target, which gives a total number of angular samples of 442 per revolution and the revolution time is $1.67 \mathrm{~s}$. Each chirp consists of 1024 samples, with the total number of samples per revolution being $\sim 28.96$ million. To scan mechanically, an existing turntable with slip rings and absolute encoder was employed. An Adlink 7200 DIO card is used to read the absolute encoder values at every CPI interval. A dedicated quadcore Dell PC is used to control the radar operation. The real time control code is written in $\mathrm{C}$ in the LabWindows/CVI platform, using multithreading to parallelize data acquisition, data processing and data display. The multithreading is necessary to ensure that all the processing can occur within a single revolution time so that there is no lag from frame to frame. The mechanical assembly of the radar system was designed in CAD and all mechanical parts were fabricated in house. The assembled system is shown in Fig. 3.

\section{Radar performance test}

The chirp bandwidth was set to be $50 \mathrm{MHz}$ to give a range bin width of $1 \mathrm{~m}$ and $512 \mathrm{~m}$ maximum instrumented range. The maximum unambiguous Doppler is $\pm 16 \mathrm{~ms}^{-1}$. To verify the link budget, a calibration was performed by operating the radar in staring mode with the radar elevation angle set to $12.5^{\circ}$ and measuring the return signal from a reference target as a function 


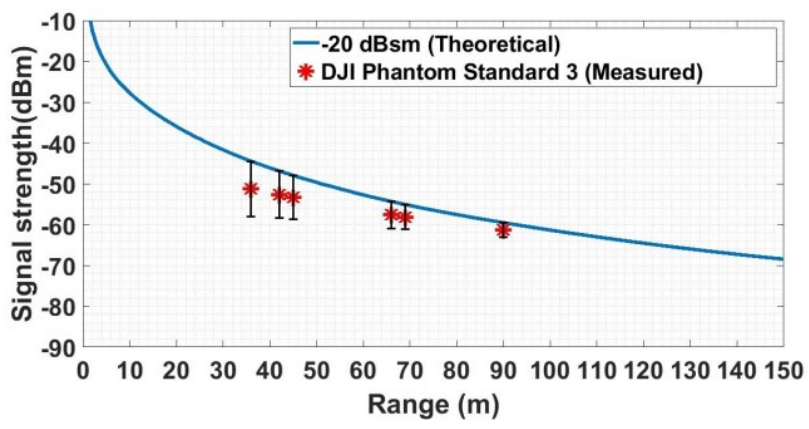

Figure 4. Calibration curve of FAROS-E
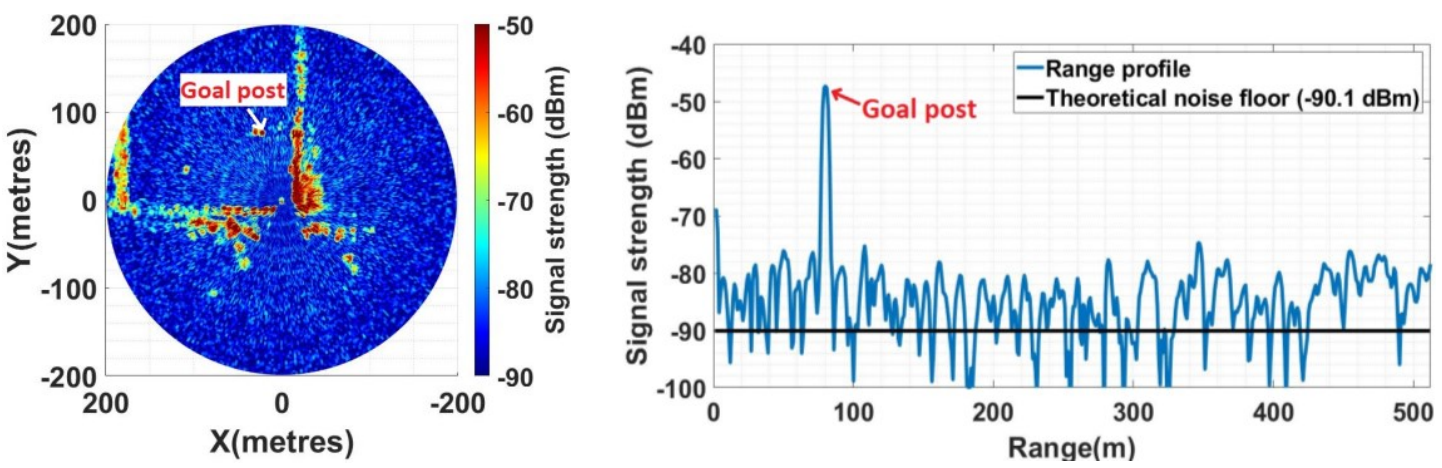

Figure 5. PPI plot (zoomed in to first $200 \mathrm{~m}$ ) or sports field showing the goal post fixed target at $80 \mathrm{~m}$ range (top) and the radar range profile showing the goal post return and the noise floor (bottom)

of range. Due to the wide elevation beamwidth, a hovering DJI Phantom Standard 3 was used instead of a tripod-mounted trihedral to position the target well above ground clutter. The RCS of this drone has previously been measured at $94 \mathrm{GHz}$ with a modal value of $-20 \mathrm{dBsm}$ [12] and for $77 \mathrm{GHz}$ we have assumed the same value. Data were collected with the drone hovering at various ranges along the elevation boresight angle and histogram plots were generated to calculate the measured modal RCS at each range. Fig. 4 is a plot of received power versus range and shows the theoretical calibration curve derived from the known radar parameters and the assumed RCS of the drone alongside the measured modal values. It can be seen that good agreement was achieved between the predicted and measured values during calibration. The measured values were $4.3 \mathrm{~dB}$ below the prediction, on average. This is acceptable as the drone was not a proper calibration target (like a trihedral). It is highly likely that there was some human error in exact antenna pointing whilst the drone was hovering, especially at close ranges. Fig. 5 (top) shows an example PPI, truncated to $200 \mathrm{~m}$ range, obtained with FAROS-E positioned in the corner of a sports field bounded to the bottom by a fence and a road, and to each side by lines of trees. The plot shows good detail in the scene, a flat noise floor and just a few low level concentric ring artefacts at close range. To observe the noise performance of the radar, a range profile taken from a chirp (line of sight) that contains a fixed, bright target (the top of a rugby goal post), is shown in Fig. 5 (bottom). It can be clearly seen that the goal post produces a very narrow, transform limited, peak in the range profile with no significant broadening right down to the noise floor over $30 \mathrm{~dB}$ below. The PPI also shows that other very bright target such as trees at close range do not cause any radial increase in the noise floor as would be attributed to significant transmitter phase noise. This confirms that FAROS-E is a very low phase noise design, which is important for the drone detection application when one is trying to detect small targets in the presence of bright targets. The low phase noise architecture follows that of an earlier very low phase noise $94 \mathrm{GHz}$ radar called T-220 [13]. 


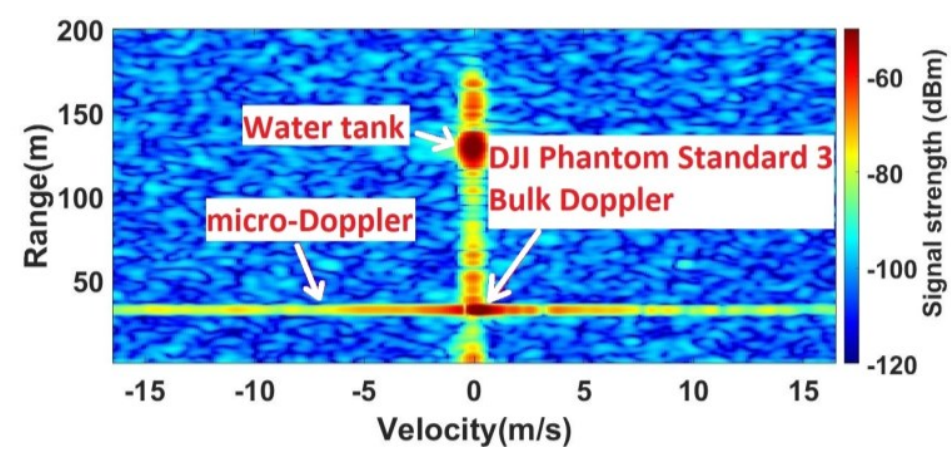

Figure 6. Range-Doppler plot from FAROS-E of a DJI Phantom Standard 3 hovering at $40 \mathrm{~m}$, producing a strong micro-Doppler signature.

Fig. 5 (bottom) confirms the noise floor is quite flat throughout the range profile right out to $512 \mathrm{~m}$. The average noise floor is $2-3 \mathrm{~dB}$ higher than the predicted value of $-90 \mathrm{dBm}$. At first, this was thought to be due to slip ring interference but that was subsequently ruled out. The reason for the discrepancy has yet to be resolved. To demonstrate the Doppler performance, a range-Doppler plot is shown in Fig. 6, in which the drone is hovering at $40 \mathrm{~m}$ range, showing a very strong micro-Doppler signature that can be used for target classification. It can be seen that the propeller micro-Doppler signature is $\sim 20 \mathrm{~dB}$ lower than the bulk Doppler in this case but still well above the noise floor.
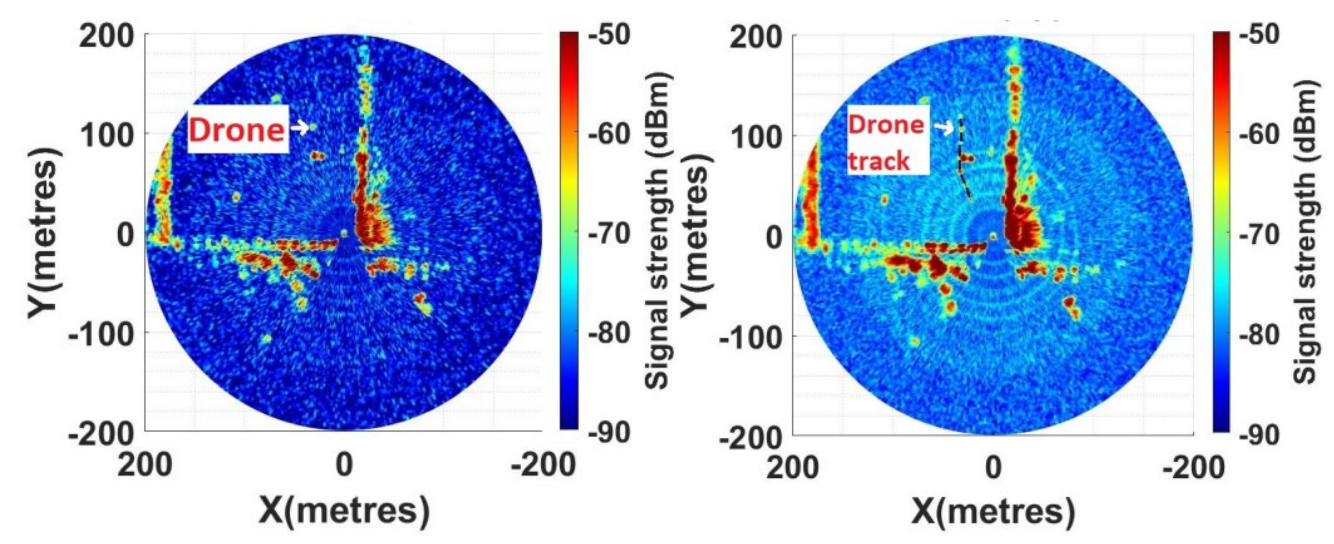

Figure 7. FAROS-E PPI plot (zoomed in) showing the Phantom detected at $\sim 110 \mathrm{~m}$ range (left) and the track of the drone obtained by combining 7 consecutive scans using maximum hold (right).

\section{Experimental trial data}

An experimental trial was conducted at one of the sports grounds of the University of St Andrews in which FAROS-E gathered $360^{\circ}$ PPI data whilst the Phantom flew around. Fig. 7 (left) shows a single PPI in which the Phantom is detected at $\sim 110 \mathrm{~m}$ range with $\sim 26 \mathrm{~dB}$ signal to noise ratio. Fig. 7 (right) shows a maximum hold combination of 7 successive PPIs revealing the drone flight path. The noise floor appears higher due to the maximum hold function. Over a series of flights at different altitudes, analysis of the data showed that the DJI Phantom 3 Standard could be detected up to ranges of approximately $300 \mathrm{~m}$, based on the magnitude of the return signal. The received power from the target was consistent with predictions obtained from the calibration curve and accounting for the hardware parameters.

An algorithm to classify the presence or absence of a drone based on the propeller microDoppler signatures was developed and implemented in real time on the radar PC. Details of the algorithm are commercial in confidence so cannot be described in detail. A specific and powerful aspect of the algorithm is that it uses the presence of propeller micro-Doppler as the 
primary method of target detection and hence is capable of detecting and classifying drones which are hovering and have zero bulk Doppler velocity. As the micro-Doppler classification relies on signal returns from the propellers which have a much smaller RCS than the fuselage the maximum classification range is typically less than the raw detection range. In trials, the algorithm was found to be capable of classifying the presence of a drone (a DJI Phantom 3 Standard) in real time at ranges up to approximately $150 \mathrm{~m}$.

\section{Conclusion}

This paper has provided a detailed description of the purpose built, FAROS-E millimeter wave $360^{\circ}$ scanning radar designed for real time drone detection and classification. The radar has excellent hardware performance with low phase noise, a flat noise floor and a well-calibrated range response. The resulting Doppler data is high fidelity and suitable for classification. Raw detection, based on received signal strength, has detected a DJI Phantom 3 Standard out to $\sim 300$ $\mathrm{m}$. A micro-Doppler based classification algorithm has been developed and was shown in trials to be capable of classifying a DJI Phantom 3 Standard at ranges up to $\sim 150 \mathrm{~m}$. The results presented here show the strong potential of a millimeter wave radar system which could be produced commercially for drone security applications.

\section{References}

[1] "Security Experts Say Drones Pose a National Security Threat | Time." [Online]. Available: https://time.com/5295586/drones-threat/. [Accessed: 02-Mar-2020].

[2] V. C. Chen, The micro-doppler effect in radar. Artech House, 2011.

[3] V. C. C. Chen, D. T. Tahmoush, and W. J. M. Miceli, Eds., Radar Micro-Doppler Signatures: Processing and Applications. Institution of Engineering and Technology, 2014.

[4] J. de Wit, "Micro-Doppler analysis of small UAVs," in 2012 9th European Radar Conference : 31 October - 2 November 2012, Amsterdam, the Netherlands, 2012, pp. 210-213.

[5] P. Molchanov, R. I. A. Harmanny, J. J. M. de Wit, K. Egiazarian, and J. Astola, "Classification of small UAVs and birds by micro-Doppler signatures," Int. J. Microw. Wirel. Technol., vol. 6, no. 3-4, pp. 435-444, 2014.

[6] M. Ritchie, F. Fioranelli, H. Griffiths, and B. Torvik, "Monostatic and bistatic radar measurements of birds and micro-drone," in 2016 IEEE Radar Conference (RadarConf), 2016, pp. $1-5$.

[7] M. Jian, Z. Lu, and V. C. Chen, "Experimental study on radar micro-Doppler signatures of unmanned aerial vehicles," in 2017 IEEE Radar Conference (RadarConf), 2017, pp. 0854-0857.

[8] "Elvira Anti Drone System - 360 Coverage and more.. | Robin Radar." [Online]. Available: https://www.robinradar.com/elvira-anti-drone-system. [Accessed: 04-Jan-2021].

[9] "SQUIRE Ground Surveillance Radar | Thales Group." [Online]. Available: https://www.thalesgroup.com/en/squire-ground-surveillance-radar. [Accessed: 04-Jan-2021].

[10] S. Rahman and D. A. Robertson, "Millimeter-wave micro-Doppler measurements of small UAVs," in Proc. SPIE 10188, Radar Sensor Technology XXI, 2017, vol. 10188, p. 101880T.

[11] "Radar \& Imaging - Farran Technology." [Online]. Available: https://www.farran.com/77-ghzfront-end--fmcw/. [Accessed: 04-Jan-2021].

[12] S. Rahman and D. A. Robertson, "In-flight RCS measurements of drones and birds at K-band and W-band," IET Radar, Sonar Navig., vol. 13, no. 2, pp. 300-309, Sep. 2018.

[13] D. A. Robertson, G. M. Brooker, and P. D. L. Beasley, "Very low-phase noise, coherent 94GHz radar for micro-Doppler and vibrometry studies," in Proc. SPIE 9077, Radar Sensor Technology XVIII, 2014, vol. 9077, p. 907719. 